\title{
What Is the Role of Hand-Assisted Laparoscopic Surgery in the Single-Port Surgery Era?
}

\author{
Chang-Nam Kim \\ Department of Surgery, Eulji University School of Medicine, Daejeon, Korea
}

See Article on Page 225-230

The paradigm of minimally invasive surgery is rapidly changing. Many surgeons feel attracted to smaller incisions, so surgery with smaller incisions is becoming the preference of many surgeons. Therefore, some surgeons are drawn to laparoscopic or robotic single-port surgery. When this is considered, hand-assisted laparoscopic surgery (HALS) seems to be unattractive because it needs a longer incision than single-port surgery and standard laparoscopic surgery (SLS). Much debate exists about the identity of HALS in terms of minimally invasive surgery. Many surgeons deny that HALS is minimally invasive surgery while some surgeons prefer to perform HALS. I think that the most important reason many surgeons deny HALS as being minimally invasive surgery is the lack of clinical experience with HALS in colectomies. Targarona et al. [1] reported a prospective randomized trial comparing SLS and HALS in colectomies. HALS was associated with a significantly greater increase in C-reactive protein (CRP) and interleukin- 6 than SLS. However, the postoperative clinical outcomes were similar for the two procedures. In my personal experience, HALS has a nearly similar postoperative clinical course as SLS except that HALS has more pain at the main incision for the hand-port placement.

Samalavicius et al. [2] did not use HALS for a right hemicolectomy because they did not see much advantage because anastomosis was performed extracorporeally. To surgeons who perform HALS, this decision is generally unusual, so that decision was possibly the authors' preference. Whether anastomosis is done intracorporeally or extracorporeally does not seem to be an impor-

Correspondence to: Chang-Nam Kim, M.D.

Department of Surgery, Eulji University Hospital, Eulji University School of

Medicine, 95 Dunsanseo-ro, Seo-gu, Daejeon 302-799, Korea

Tel: +82-42-259-1335, Fax: +82-42-259-1335

E-mail: kimcn@eulji.ac.kr

(c) 2013 The Korean Society of Coloproctology

This is an open-access article distributed under the terms of the Creative Commons Attribution NonCommercial License (http://creativecommons.org/licenses/by-nc/3.0) which permits unrestricted noncommercial use, distribution, and reproduction in any medium, provided the original work is properly cited. tant issue in terms of the adoption of HALS. I feel HALS is very beneficial for some difficult cases of a right colectomy. Because of this, HALS is more difficult to standardize than SLS. The most important reason is that the location of the hand-port is mainly influenced by the operator's dominant hand: right-handed or lefthanded. According to the operator's dominant hand, the locations of the hand- and the camera-port placements are different. For surgeons who are ambidexterous, HALS can be more easily adopted and may be a more powerful instrument than for the surgeons who have one dominant hand. Ballantyne and Leahy [3] mentioned that HALS is an adjunct and a bridge towards total laparoscopic surgery. However, my idea is something different from that. HALS seems not to be an adjunct or bridge towards SLS, but to be a unique type of surgery that has its own surgical indications, including bulky cancer, adhesion, ileus, and the need for time-consuming surgery like a total colectomy or a total proctocolectomy.

Nowadays, young surgeons are very confused about what kind of surgery should be performed for a colectomy. Of course, young surgeons are very attracted to operations needing smaller incisions, like single-port surgery. In this era, surgeons should strictly evaluate their surgical abilities and the characteristics of both the tumor and the patient. Is the surgery for the surgeon's benefit or for the patient's? Sometimes, surgeons easily decide to employ their preferred type of surgery. In spite of that tendency, surgeons should absolutely focus on the characteristics of the tumor and the patient: tumor size, adhesion, ileus, invasion to adjacent organs, and obesity. Even to experienced laparoscopic surgeons, laparoscopic colectomies for obese patients are generally difficult to perform. Heneghan et al. [4] compared HALS and SLS performed on obese patients (body mass index $>30 \mathrm{~kg} / \mathrm{m}^{2}$ ). They showed that the length of stay and the morbidity and mortality rates were comparable between the two groups. However, conversions to open surgery occurred less often in HALS than in SLS (3.5\% vs $12.7 \%, \mathrm{P}=0.014)$. Therefore, HALS may be an important option for a colectomy in obese patients.

Conclusively, HALS seems to be a unique genre of minimally invasive surgery that, in some cases, can be performed instead of SLS and open surgery. Adoption of minimally invasive surgery 


\section{Coloproctology

should absolutely be based on the characteristics of the tumor and the patient. If surgeons do not follow this principle, they may inappropriately select minimally invasive surgery for some colorectal cancer patients.

\section{REFERENCES}

1. Targarona EM, Gracia E, Garriga J, Martínez-Bru C, Cortes M, Boluda R, et al. Prospective randomized trial comparing conventional laparoscopic colectomy with hand-assisted laparoscopic colectomy: applicability, immediate clinical outcome, inflammatory response, and cost. Surg Endosc 2002;16:234-9.
2. Samalavicius NE, Gupta RK, Dulskas A, Kazanavicius D, Petrulis K, Lunevicius R. Clinical outcomes of 103 hand-assisted laparoscopic surgeries for left-sided colon and rectal cancer: single institutional review. Ann Coloproctol 2013;29:225-30.

3. Ballantyne GH, Leahy PF. Hand-assisted laparoscopic colectomy: evolution to a clinically useful technique. Dis Colon Rectum 2004;47:753-65.

4. Heneghan HM, Martin ST, Kiran RP, Khoury W, Stocchi L, Remzi $\mathrm{FH}$, et al. Laparoscopic colorectal surgery for obese patients: decreased conversions with the hand-assisted technique. J Gastrointest Surg 2013;17:548-54. 